\title{
Early Outcome of Off-pump Coronary Artery Bypass Graft Surgery in Patients with Multivessel Coronary Artery Disease
}

N U Ahmed ${ }^{1}$, K Hasan ${ }^{2}$, S K Raha ${ }^{3}$

\section{Abstract:}

As there have been appreciable bodies of evidence supporting the theoretical and practical advantages of off-pump coronary artery bypass grafting (OPCAB) over the conventional coronary artery bypass graft $(C A B G)$ to avoid the harmful effects of cardiopulmonary bypass $(C P B)$, many cardiac surgeons are using $O P C A B$ as an effective alternative to conventional CABG. This study performed in National Institute of Cardiovascular Diseases (NICVD) evaluated the early surgical outcomes of $O P C A B$ in terms of mortality and major post-operative morbidities and compared them with that of conventional CABG. Total 120 patients with multivessel coronary artery disease were allocated into two groups: a) 60 patients who underwent $O P C A B$ and b) another 60 patients who underwent conventional CABG between January 2009 and December 2011. The sex and mean ageA reduced level or complete cessation of sweating can be caused by a variety of factors affecting sweat glands directly or indirectly through alterations in their nerve supply. The most common presentation is the syndrome of heat intolerance with or without features of dysautonomia. An acquired idiopathic form of generalized anhidrosis is characterized by loss of sweating in the absence of any neurological features or destruction of sweat glands. I Idiopathic acquired generalized anhidrosis is a very rare condition in which the pathogenesis is still unknown. ${ }^{2}$ Cholinergic urticaria has been associated with some cases of this acquired idiopathic form of generalized anhidrosis. ${ }^{3}$ distributions of patients were similar among two groups. Smoking, diabetes mellitus, hypertension and dyslipidemia were major risk factors. All co-morbid conditions were homogenously distributed between the two groups. Majority of the patients had triple vessel disease. Nearly three-quarter (73.3\%) of patients in OPCAB group and $80 \%$ in conventional $C A B G$ group received 3 grafts $(p=0.470)$. The mean total operative time (258 \pm 39.5 minutes versus $306 \pm 44.8$ minutes; $p<0.001)$, intubation times $(7.6 \pm 0.3$ versus $16.3 \pm 0.5$ hours; $p<0.001)$, blood losses $(377.8 \pm 22.3 \mathrm{ml} v s .602 .0 \pm 18.9 \mathrm{ml}, p<0.001)$; requirements

1. Dr. Nasir Uddin Ahmed, Former Professor and Head, Department of Cardio-Vascular Surgery, NICVD, Dhaka.

2. Dr. Kamrul Hasan, Associate Professor, Department of Cardiac Surgery, NICVD, Dhaka.

3. Dr. Sanjay Kumar Raha, Assistant Registrar, Department of Cardiac Surgery, NICVD, Dhaka.

Corresponding author: Dr. Nasir Uddin Ahmed, Former Professor and Head, Department of Cardio-Vascular Surgery, NICVD, Dhaka. for blood and blood products (689.7 \pm 21.1 vs. $1199.3 \pm 34.5$ $\mathrm{ml}, p<0.0010)$; intensive care unit stays $(31.7 \pm 0.9$ hours versus $41.6 \pm 1.5$ hours; $p<0.001)$ and hospital stays $(6.2 \pm 0.2$ days versus $8.3 \pm 0.3$ days; $p<0.0010)$ were all significantly shorter in the $O P C A B$ group. $O P C A B$ is a safe and effective procedure for patients with multivessel coronary artery disease and is associated with reduced morbidity and mortality. However, large randomized studies with long-term follow-up may show the real benefits of OPCAB compared with $C A B G$ on $C P B$.

Introduction:

Jones et al. (1996) examined seven large datasets, with more than 172,000 patients undergoing isolated CABG, to assess the predictive power of certain preoperative values on operative mortality. Seven variables were found to be predictive of mortality in all datasets. These seven core variables included urgency of operation, age, prior heart surgery, gender, left ventricular ejection fraction (LVEF), and percentage of stenosis of the left main coronary artery, and number of major coronary arteries with $70 \%$ stenosis.

$\mathrm{CABG}$ with cardioplegia has been considered the gold standard operation for coronary revascularization. The introduction of the heart-lung machine created optimal operative conditions of bloodless, motionless operative field and allowed CABG to be done consistently with generally good results in most patients (Favaloro, 1970). However, this technique is linked to several side effects mostly due to use of aortic cross clamp, cardioplegia, and cardiopulmonary bypass. High risk patients are extremely sensitive to cardioplegic arrest and have higher intra-operative and post-operative risk (Wan, et al., 2004).

Contacts of blood components with the artificial surfaces of bypass circuit, aortic cross-clamping and reperfusion injury are considered the main causative factors of inflammatory response following cardiac surgery. Furthermore hypothermia, duration of CPB, hypoperfusion, and microemboli of gaseous or particulate nature all contribute to end organ injury (Angelini, et al,, 2003). The damaging effects on platelets and clotting factors and higher doses of heparin required, contribute to the increased need for blood and blood product transfusion with traditional CABG (Bowles, et al., 2001).

Atrial fibrillation occurs in $20-40 \%$ of patients who had CABG with cardiopulmonary bypass and is associated with higher risk of cerebrovascular accidents (Altarabsheh, et al., 2009). Global ischemia, manipulation of the right atrium and the inflammatory response which occur with cardiopulmonary bypass may result in lung and renal injuries with the resulting disturbances of acid-base balance and electrolytes may produce atrial irritability leading to atrial fibrillation (Altarabsheh, et al., 2009). 
The higher incidence of post-operative IABP insertion, renal hemodialysis, mechanical ventilation and/or reintubation or tracheostomy could be responsible for the significant higher rate of readmission for CPB patients (Al-Ruzzeh. et al., 2003).

The invention of $\mathrm{OPCAB}$ is often attributed to Kolessov in 1967 (Mack, 2004). In the mid-1990s interest in beating-heart techniques experienced resurgence in an attempt to decrease the morbidity associated with $\mathrm{CABG}$ without jeopardizing the benefits (Mack, 2004). Now OPCAB is widely accepted and considered to be safe for myocardial revascularization specially for high risk patients (Takai, et al., 2006).

With appropriate modern retractor-stabilizers, heart positioning devices, techniques of exposure of all surfaces of heart, intracoronary shunts, and adequate surgeon experience, similar completeness of revascularization and graft patency can be achieved with OPCAB (Cohn, 2008).

The rapidly increasing incidence of diabetes mellitus, hypertension, and hypercholesterolemia in most communities has given rise to more severe and diffuse coronary artery disease (Fouda, et al., 2007). As a result of improvement in invasive cardiology most patients referred for $\mathrm{CABG}$ have diffuse disease and poor ventricles. The global ischemia caused by conventional $\mathrm{CABG}$ could be detrimental to them. The OPCAB technique was developed with specific purpose of reducing mortality and morbidity in high risk patients (Mack, et al., 2004).

The shorter operating time in OPCAB group is probably because of the shorter time required for hemostasis and no time spent on cannulation, managing cardiac arrest and rewarming from hypothermia during CPB. There is also less intra-operative and post-operative blood loss, a lower incidence of re-exploration for bleeding, a smaller transfusion volume and lower incidence of transfusion (Ishida, et al., 2002).

The shorter ICU stay and ventilation time in the OPCAB group may be related to lack of the pulmonary dysfunction that occurs after $\mathrm{CPB}$. Glomerular filtration rate and renal tubular function are better protected by OPCAB group than by conventional CABG (Ishida, et al., 2002).

There are also potential economic benefits including reduced postoperative patient instability and faster recovery rate, reduced use of vasoactive drugs, less need for blood products and cost effective resource utilization (Royse, et al., 2003).

\section{Methodology:}

This was a prospective non-randomized clinical study done in National Institute of Cardiovascular Diseases (NICVD) from January 2009 to December 2011. Among the 120 patients with multivessel coronary artery disease 60 patients underwent OPCAB (Group: A) and the rest 60 underwent conventional CABG (Group: B). Data of different preoperative, peroperative and postoperative variables collected by interview schedule and checklist were evaluated and compared.

\section{Anesthesia and Monitoring:}

Patients were premedicated with morphine $(0.1 \mathrm{mg} / \mathrm{kg})$ and bromazepam (3mg). Induction was achieved with midazolam $(15-20 ? \mathrm{~g} / \mathrm{kg})$ and fentanyl $(10-100 ? \mathrm{~g} / \mathrm{kg})$. Muscle relaxation was achieved with vecuronium bromide $(0.10-15 \mathrm{mg} / \mathrm{kg})$. Anesthesia was maintained with oxygen, air, and incremental doses of midazolam and fentanyl. All patients had a radial artery cannula and a central venous catheter for the monitoring of mean arterial pressure, central venous pressure. Femoral artery cannulation was performed in patients with poor ventricular function (left ventricular ejection fraction $<30 \%$ ) in the event that urgent institution of an intra-aortic balloon pump was required. A combination of lead II and V5 was continuously displayed and used for ST-segment analysis. Arterial blood gases and activated clotting time were monitored every 30 minutes. The patient's temperature, which was constantly monitored by a properly placed nasal temperature probe, was maintained close to 36 ? $\mathrm{C}$ with a water-heated mattress, a fluid warmer, and an ambient operating room temperature of $>20$ ? C..

\section{Technique of OPCAB:}

All patients were operated on through a median sternotomy. The internal thoracic artery, the radial artery, and the saphenous vein were harvested as appropriate with standard techniques. After harvesting the arterial and venous conduits patients were given heparin $(100 \mathrm{IU} / \mathrm{kg}$ ) before starting distal anastomoses. Regional myocardial immobilization was achieved with a suction stabilizer (Octopus) and apical suction device (Star Fish). Intracoronary shunts of different sizes $(1.5 \mathrm{~mm}, 2 \mathrm{~mm}$, and $2.5 \mathrm{~mm}$ ) were used in most patients to maintain coronary flow, thereby reducing myocardial ischemia and at the same time minimizing bleeding from the coronary arteriotomy. The left anterior descending (LAD) artery was revascularized first using left internal mammary/ thoracic artery (LIMA/ LITA). Visualization was enhanced by using a humidified blower and normal saline spray. Proximal anastomoses were performed on the partially clamped ascending aorta. Occluded coronary arteries were usually revascularized before stenosed ones. However, the sequence of grafting was individualized for each patient and depended on various factors, including hemodynamic stability after tilting the heart and the severity of the stenosis. Distal anastomoses were performed with continuous 7-0 or 8-0 polypropylene (Prolene) monofilament suture. Proximal anastomoses were performed with 6-0 continuous Prolene suture. Before placing the side biting clamp for the proximal anastomosis, the systemic blood pressure was lowered, either by the anesthesiologist using antihypertensive agents or by the surgeon using inflow occlusion. For exposure of the obtuse marginal vessel, 2 or 3 deep pericardial traction sutures were positioned between the left superior pulmonary vein and the inferior vena cava. The other measures that were applied for exposure and maintenance of better hemodynamic included extensive right pleurotomy, deep 
vertical right pericardiotomy, and hemisternum elevation. After the procedure, heparin therapy was reversed with protamine sulfate in a $1: 1$ ratio. The leg, forearm, and chest wounds were closed and the patients were shifted to ICU. Total operation time and number of grafts were recorded.

Technique of Coronary Artery Bypass on CPB:

After median sternotomy standard CPB was established with ascending aortic and 2 -stage venous cannulation. Conduits were harvested and prepared, and the patients were heparinized with an initial dose of $300 \mathrm{IU} / \mathrm{kg}$ heparin to achieve a target activated clotting time greater than 450 second. The blood pressure was maintained within 50 to 70 $\mathrm{mm} \mathrm{Hg}$. The systemic temperature was kept around $32^{\circ} \mathrm{C}$. The aorta was cross-clumped, and myocardial protection was achieved with intermittent antegrade and retrograde hyperkalemic cold blood cardioplegia according to a standard protocol in all cases. After the induction of ischemic arrest for 3 minutes, subsequent doses were administered every 15-20 minutes. The distal anastomoses were performed first precisely on the motionless and bloodless field. On completion of all the distal anastomoses aortic clamp was released and the proximal anastomoses were performed with partial clamping of the ascending aorta during rewarming. Then the patients were gradually weaned from cardiopulmonary bypass (CPB). Heparin neutralization was done by protamine in $1: 1$ ratio at the end of the procedure. The leg, forearm, and chest wounds were closed and the patients were shifted to ICU. Total operation time and number of grafts were recorded.

\section{Postoperative Management:}

After surgery, all patients were admitted to the intensive care unit (ICU). In ICU cardiac function was monitored by using both invasive and noninvasive methods. Respiratory, renal function and hourly blood loss were monitored meticulously. Extubation was done as early as possible while the patients fulfill the extubation criteria. Arterial blood gas, serum electrolytes and hematocrit estimation were done as per standard protocol. During immediate post-operative period intravenous nitroglycerine was used as coronary vasodilator. Systemic hypertension was treated also with nitroglycerine and on rare occasion sodium nitroprussidé was used. Systemic hypotension was treated with volume replacement and inotropic support. Post-operative complications were identified and treated accordingly if developed. Pre-operative medications continued as per protocol. Patients were encouraged to use intensive spirometry and early mobilization. Then the patients were shifted to general wards and subsequently discharged according to unit protocol. Antibiotic administration was continued until the patient had central lines or chest drains (normally 24-48 hours).

\section{Result:}

Patient characteristics of multivessel coronary artery disease were shown in the Table-1. The mean age of the study was 58.4?7.8 years for the OPCAB group and $58.2 \pm 7.2$ years for on-pump CABG group with no significant difference of age distribution between two groups.
Table-1: Patient characteristics of multivessel coronary artery disease*

\begin{tabular}{|c|c|c|c|}
\hline Variables & $\begin{array}{l}\text { OPCAB group } \\
(n=60)\end{array}$ & $\begin{array}{l}\text { CCAB group } \\
(n=60)\end{array}$ & $p$ \\
\hline Age, years & $58.4 \pm 7.8$ & $58.2 \pm 7.2$ & 0.91 \\
\hline Male, $n(\%)$ & $54(90)$ & $52(86.7)$ & 0.500 \\
\hline Female, $n(\%)$ & $6(10)$ & $8(13,3)$ & 0.500 \\
\hline $\mathrm{BMI}\left(\mathrm{kg} / \mathrm{m}^{2}\right)$ & $26.1 \pm 3.3$ & $26.5 \pm 6.2$ & 0.426 \\
\hline Hypertension, n (\%) & $32(53.3)$ & $30(50.0)$ & 0.796 \\
\hline Diabetes, $n(\%)$ & $20(33.3)$ & $20(33.3)$ & 0.608 \\
\hline Smoking, n (\%) & $40(66.7)$ & $38(63.3)$ & 0.787 \\
\hline Dyslipidemia, n (\%) & $38(63.3)$ & $32(53.3)$ & 0.432 \\
\hline Family H/O CAD, n (\%) & $6(10.0)$ & $8(13,3)$ & 0.500 \\
\hline Past H/O CVA, n (\%) & $4(6.7)$ & $6(10.0)$ & 0.500 \\
\hline $\mathrm{COPD}, \mathrm{n}(\%)$ & $6(10.0)$ & $6(10.0)$ & 0.665 \\
\hline History of MI, n (\%) & $12(20.0)$ & $14(23.3)$ & 0.754 \\
\hline PVD, $n(\%)$ & $6(10.0)$ & $6(10.0)$ & 0.665 \\
\hline Renal dysfunction, $\mathrm{n}(\%)$ & $4(6.7)$ & $2(3.3)$ & 0.500 \\
\hline Arrhythmia, n (\%) & $4(6.7)$ & $4(6.7)$ & 0.694 \\
\hline $\operatorname{LVEF}(\%)$ & $54.1 \pm 2.4$ & $53.3 \pm 1.8$ & 0.801 \\
\hline NYHA class III or IV, n $(\%)$ & $10(16.67 \%)$ & $8(13.33 \%)$ & 0.952 \\
\hline $\begin{array}{l}\text { CCS angina class III or IV, } \mathrm{n} \\
(\%)\end{array}$ & $26(43.33 \%)$ & $32(53.33)$ & 0.757 \\
\hline DVD & $18(30.0)$ & $10(16.7)$ & 0.222 \\
\hline TVD & $42(70.0)$ & $50(83.3)$ & 0.222 \\
\hline
\end{tabular}

Data are presented as the mean ? SD where appropriate. OPCAB: Off-pump coronary artery bypass; CCAB: Conventional coronary artery bypass; COPD: Chronic obstructive pulmonary disease; CVA: Cerebrovascular accident; MI: Myocardial infarction; NYHA: New York Heart Association; CCS: Canadian Cardiovascular Society angina class; PVD: Peripheral vascular disease.

In our study both sexes were homogenously distributed between OPCAB and on-pump groups. But male predominance in this series was similar to other national and international studies Among the 60 patients, OPCAB group had $27(90 \%)$ and on-pump group had $26(86.7 \%)$ male patients. These findings suggest that atherosclerotic coronary artery disease is more prevalent among male. In our study overweight and obese patients were higher among conventional CABG group than that in Off-pump CABG group (43.3\% vs. 33.3\%). The mean BMI was almost similar between groups (26.1 ? 3.3 vs. 26.5 ? 6.2 $\mathrm{kg} / \mathrm{m} 2, \mathrm{p}=0.955$ ). In our study the commonest co-morbid factor was smoking in both groups $(66.7 \%$ in OPCAB group vs. $63.3 \%$ in on-pump group). It was followed by dyslipidemia ( $63.3 \%$ vs. $53.3 \%$ ), hypertension $(53.3 \%$ vs. $50 \%$ ) and diabetes mellitus (33.3\% in both groups). Other 
co-morbid factors were family history of CAD (10\% vs. $13.3 \%)$, past history of CVA $(6.7 \%$ vs. $10 \%)$, COPD $(10 \%$ vs. $10 \%)$, history of MI ( $20 \%$ vs. $23.3 \%)$, PVD ( $10 \%$ vs. $10 \%)$ and renal dysfunction $(6.7 \%$ vs. $3.3 \%)$. All were almost identically distributed between the groups ( $p>$ 0.05 ). In our study the mean left ventricular ejection fraction (LVEF) was almost similar in both groups (54.1 ? $2.4 \%$ vs. 53.3 ? $1.8 \%, \mathrm{p}=0.801)$. Pre-operative angiographic study demonstrated that in our study majority of the patients had triple vessel disease (TVD) in each group. OPCAB group has $21(70 \%)$ and on-pump group has $25(83.3 \%)$ patients with TVD. On the other hand, 9 $(30 \%)$ patients in OPCAB group and $5(16.7 \%)$ patients of on-pump group had double vessel disease (DVD). The time needed for operation was significantly higher in patients who underwent conventional CABG compared to patients off-pump CABG (306.3 \pm 44.8 vs. $258.6 \pm 39.5$ minutes, $p$ $<0.001)$. The mean cardiopulmonary bypass $(\mathrm{CPB})$ time was $191.1 \pm 55.9$ minutes in conventional CABG group. Nearly three-quarter $(73.3 \%)$ of patients in OPCAB group $80 \%$ in conventional $\mathrm{CABG}$ group required 3 grafts $(\mathrm{p}=$ $0.470) .2(6.7 \%)$ on-pump patients and $1(3.3 \%)$ OPCAB patients had 4 distal anastomoses. On the other hand, none in on -pump group but $1(3.3 \%)$ OPCAB patient had single graft. There was no significant difference between the groups in terms of distal anastomosis. Several post-operative variables had been compared between the groups in our study such as mean ventilation time, prolonged inotropic support, use of IABP, total bleeding, amount of blood products needed, length of ICU stay, length of hospital stay, and 30-days mortality. In our study the mean ventilation time was significantly higher in the on-pump CABG group than those in off-pump CABG group ( $16.3 \pm 0.5$ hours vs. $7.6 \pm 0.3$ hours, $p<0.001)$. No patient in our study required prolonged ventilation $(>24$ hours). In NICVD there is a trend to use inotropic support to almost all post-CABG patients unless otherwise specified. So, we used prolonged post-operative inotropic support ( $>24$ hours) or use of IABP as post-operative outcome variables. In our study one (3.3\%) on-pump patient required prolonged inotropic support and another one $(3.3 \%)$ required IABP but none of the OPCAB patients received prolonged inotropic support or IABP.

Table 2: Intraoperative Variables*

$\begin{array}{llll}\text { Variables } & \begin{array}{l}\text { OPCAB group } \\ \text { (n=60) }\end{array} & \begin{array}{l}\text { CCAB group } \\ (\mathbf{n = 6 0 )}\end{array} & \boldsymbol{p} \\ \begin{array}{l}\text { Conversion to CPB, n (\%) } \\ \text { CPB time, min }\end{array} & 2(3.33 \%) & 191.1 \pm 55.9 & \\ \begin{array}{l}\text { Total operating time, min } \\ \text { Conduit used }\end{array} & 258.6 \pm 39.5 & 306.3 \pm 44.8 & <0.001 \\ \quad \text { LIMA, n (\%) } & 60(100 \%) & 60(100 \%) & 0.694 \\ \quad \text { Radial artery, n (\%) } & 6(10 \%) & 8(13.33 \%) & 0.500 \\ \quad \text { SVG, n (\%) } & 60(100 \%) & 60(100 \%) & 0.694 \\ \text { Graft distribution } & & & \\ \quad \text { LAD territory, n (\%) } & 60(100 \%) & 60(100 \%) & 0.694 \\ \quad \text { Circumflex territory, n (\%) } & 56(93.33 \%) & 60(100 \%) & 0.246 \\ & 51(85 \%) & 50(83.33 \%) & 0.083 \\ \text { Intraoperative IABP } & 0(0 \%) & 2(3.3 \%) & 0.500\end{array}$

Data are presented as the mean $\pm \mathrm{SD}$ where appropriate. OPCAB: Off-pump coronary artery bypass; CCAB: Conventional coronary artery bypass; $\mathrm{CPB}$ : Cardiopulmonary bypass; IABP: Intra-aortic balloon pump; LAD: Left anterior descending artery; RCA: Right coronary artery; LIMA: Left internal mammary artery; RIMA: Right internal mammary artery; SVG: Saphenous vein graft.

In our study total post-operative bleeding was significantly lower in OPCAB group than on-pump group $(377.8 \pm 22.3$ $\mathrm{ml}$ vs. $602.0 \pm 18.9 \mathrm{ml}, \mathrm{p}<0.001)$. The requirement of blood product was also significantly lower in $\mathrm{OPCAB}$ group (689.7 \pm 21.1 vs. $1199.3 \pm 34.5 \mathrm{ml}, \mathrm{p}<0.0010)$. In this study, the average ICU-stay period in OPCAB group was shorter (31.7 \pm 0.9 hours) than that in on-pump group $(41.6 \pm 1.5$ hours $)(p<0.001)$. Total postoperative stay (days) in hospital was also shorter in OPCAB group ( $6.2 \pm 0.2$ vs. $8.3 \pm 0.3$ days, $p<0.001)$. In our study none of the patients died within 30 days of operation in $\mathrm{OPCAB}$ group while two $(3.3 \%)$ of patients died in conventional $\mathrm{CABG}$ group $(p=0.50)$. However, it was not statistically significant.

Table 3: Comparison of post-operative outcome between groups

$\begin{array}{llll}\text { Variables } & \begin{array}{l}\text { OPCAB group } \\ (\mathbf{n}=60)\end{array} & \begin{array}{l}\text { CCAB group } \\ (\mathrm{n}=60)\end{array} & p \\ \text { 30 days mortality, } \mathrm{n}(\%) & 0(00 \%) & 2(3.3 \%) & 0.500 \\ \text { Ventilation time, hours, } \mathrm{n} & 7.6 \pm 0.3 & 16.3 \pm 0.5 & <0.001 \\ \text { Prolonged inotropic support } & 0(00 \%) & 4(6.7) & 0.246 \\ \text { Postoperative IABP } & 0(00 \%) & 2(3.3 \%) & 0.500 \\ \text { Total bleeding (ml) } & 378 \pm 12 & 602 \pm 9 & <0.001 \\ \text { Amount of blood products } & 690 \pm 21 & 1199 \pm 34 & <0.001 \\ \text { needed (ml) } & & & \\ \text { Length of ICU stay(hours) } & 31.7 \pm 0.9 & 41.6 \pm 1.5 & <0.001 \\ & & 8.3 \pm 0.3 & <0.001 \\ \text { Length of hospital stay(days) } & 6.2 \pm 0.2 & & \\ \text { Re-exploration for bleeding } & 0(00 \%) & 4(6.7) & 0.246 \\ & & & \\ \text { Stroke } & 0(00 \%) & 2(3.3 \%) & 0.500 \\ \text { Pulmonary complication } & 4(6.7) & 6(10.0) & 0.500 \\ \text { Perioperative MI } & 0(00 \%) & 2(3.3 \%) & 0.500 \\ \text { Arrhythmia } & 6(10.0) & 12(20 \%) & 0.236 \\ \text { Surgical site infection } & 4(6.7) & 6(10.0) & 0.500 \\ \text { Renal dysfunction } & 4(6.7) & 6(10.0) & 0.500\end{array}$

In the immediate postoperative period, one $(3.3 \%)$ of the on-pump patients developed new Q-wave myocardial infarction. That patient requiring IABP support died later on second postoperative day. The mean period of mechanical ventilation, amount of blood products needed, length of ICU stay and hospital stay during the early post-operative period- all were significantly greater in on-pump group as opposed to OPCAB group. All these reflect definite clinical advantage as well as favorable economic outcome associated with OPCAB group of patients. The distribution of early post-operative 
complications between the two groups was compared using appropriate statistical tests. Two $(6.7 \%)$ patients of on-pump group but neither of OPCAB group required re-exploration for bleeding $(p=0.246)$. One $(3.3 \%)$ of on-pump patient developed stroke and another one $(3.3 \%)$ developed $\mathrm{MI}$ in the on-pump group but no one developed new postoperative arrhythmias were developed in $6(20 \%)$ on-pump and $3(10 \%)$ OPCAB patients $(\mathrm{p}=0.236)$. In most (7 patients) of them there were atrial arrhythmia and the remaining ( 2 patients) had ventricular tachycardia. Pulmonary complication, infective complication and renal dysfunction were also more common in on-pump group $(10 \%$ vs. $6.7 \%$ each, $\mathrm{p}=0.500)$. In our study, pulmonary complications (total 5) were atelectasis (3 patients), pneumonia ( 2 patients), and pleural effusion ( 1 patient) who required tube thoracostomy. The infective complications (total 5) were superficial sternal wound infection (2 patients), deep sternal wound infection (1 patient) and donor site infection ( 2 patients). Thus, postoperative complications were relatively less common in OPCAB group although statistically not significant. But this might be significant if larger sample would have been taken.

\section{Discussion:}

Initial experience with $\mathrm{OPCAB}$ was limited to single or double vessel disease (Arom, et el., 2000a, Puskas, et al., 1998). With the availability of better stabilization techniques and surgeons' increasing experience, $\mathrm{OPCAB}$ is being used for patients with multivessel disease (Hernandez, et al., 2001, Roy, et al., 2001). In the present study, we have analyzed our experience with $\mathrm{OPCAB}$ in patients with multivessel disease. As in other studies we have found lower morbidity in patients who underwent OPCAB. Long-term studies comparing OPCAB and on-pump CABG are few. Although OPCAB approach has fewer short-term complications than on-pump $\mathrm{CABG}$, incomplete revascularization is more common with off-pump approach, which led to more vascular complications and repeat revascularization $(\mathrm{Hu}$, et al., 2010). Complete revascularization is believed to be important in producing a re-intervention-free result following OPCAB (Gundry, et al., 1998). Meharwal et al. (2003) showed the average numbers of grafts were $3.0 \pm 0.7$ for OPCAB group and 3.2 \pm 0.8 for on-pump group. Shroyer et al. (2009) in their study showed the average numbers of grafts were $2.9 \pm 0.9$ for OPCAB group and $3.0 \pm 1.0$ for on-pump group. Youn et al. (2007) demonstrated in their study that patients with on-pump CABG tended to have more grafts, but there was no significant difference in number of distal anastomoses and complete revascularization between the groups. However, with appropriate surgical techniques and experience complete revascularization and similar graft patency can be achieved with OPCAB. Technical improvement and experience have led some surgeons to perform off-pump total arterial grafting using two internal thoracic arteries (ITA) or one ITA and radial artery for multivessel coronary artery disease in regular basis. In our experience, complete We have used intracoronary shunts in all patients during distal coronary anastomoses. Positioning and stabilization of the heart in $\mathrm{OPCAB}$, myocardial revascularization can be achieved on beating heart (Raja. et al., 2009).

specially during circumflex and posterior descending artery anastomosis, are associated with significant haemodynamic changes (Mathison, et al., 2000). These changes may be further exacerbated by the snaring of the coronary arteries. Several studies have shown the effectiveness of intracoronary shunts for maintaining myocardial perfusion to avoid ischemia of target vessels during OPCAB, although the use of shunts is not widespread and remains controversial (Lucchetti, et al., 1999). We have found intracoronary shunts useful.

The mean period of mechanical ventilation, amount of blood products needed, length of ICU stay and hospital stay during the early post-operative period- all were significantly greater in on-pump group as opposed to OPCAB group. All these reflect definite clinical advantage as well as favorable economic outcome associated with OPCAB group of patients. Transmission of viral infections, induction of immunologic transfusion reactions, and suppression of the immune system remain important risks related to the transfusion of blood and blood products despite improvements in donor-screening methods (Consten, et al., 1996). Also OPCAB has been shown to be associated with decreased morbidity and mortality in highrisk patients, including the elderly, patients with poor left ventricular function, renal dysfunction, left main stenosis, or chronic obstructive pulmonary disease, and patients with prior neurologic dysfunction (Boyd, et al, 1999). As in many studies, hospital mortalities for off-pump and on-pump CABG have been comparable. Meharwal et al. (2003) reported that the operative mortality was higher in on-pump group $(1.86 \%$ vs. $0.97 \%, \mathrm{p}<.001)$. Ruzzeh et al. (2003) in a multi-centre comparative analysis showed similar result (1.4\% vs. $2.9 \%$ ). But, Sajja et al. (2008) $(2.8 \%$ vs.3.9\%, $=0.746)$ and $\mathrm{Hu}$ et al. (2010) [adjusted OR 0.74 $(0.46-1.18)$, at $95 \% \mathrm{CI}, \mathrm{p}=0.21]$ showed different results.

\section{Study limitations:}

The present study has several limitations and those are as follows:

1. Sample size was small and patients were selected purposefully.

2. They were not randomly assigned to either group.

3. The surgical procedure either OPCAB or on-pump $\mathrm{CABG}$ was determined by the surgeon. Therefore selection bias may affect our findings.

4. The duration of follow up of this study was limited. Clinical outcomes were restricted to 30 -days mortality. No data beyond three months follow-up were available. Nothing was mentioned about the quality of life after CABG.

5. As a single institutional study the conclusions may not be applicable in general because of differences in practice patterns of other centres.

6. Other factors such as variations in surgical skill, patient difference in extent or distribution of coronary artery disease and echocardiography reports although unavoidable should also are considered. 


\section{Recommendations:}

OPCAB surgery can yield better outcome with shorter recovery time and less adverse effects than conventional CABG. We recommend OPCAB as a safe and effective surgical strategy for the patients with multivessel coronary artery disease.

A prospective large scale multi-institutional randomized trial along with long term serial and regular follow up and evaluation of graft patency are necessary to confirm our findings and to define the long term clinical and functional results of both on-pump and off-pump CABG.

Development of well trained cardiac surgical and anesthetic team, establishment of cardiac surgical centres equipped with modern devices, adequate logistic support and research of ischemic heart disease are needed to ensure up to date service and to widen the future prospects of revascularization procedures.

\section{References:}

1. Al-Ruzzeh, S., Ambler, G., Asimakopoulos, G., Omar, R.Z., Hasan, R., Fabri, A., et al., 2003. Off-pump Coronary Artery Bypass (OPCAB) Surgery Reduces Risk-Stratified Morbidity and Mortality: A United Kingdom Multi-Centre Comparative Analysis of Early Clinical Outcome. Circulation, 108, pp. 1-8.

2. Altarabsheh, S.E., 2009. Outcomes of Off-pump Coronary Artery Bypass Surgery. Bahrain Medical Bulletin, 31, pp. 3-12.

3. Angelini, G.D., Taylor, F.C., Reeves, B.C., Ascione, R., 2003. Early and midterm outcome after off-pump and on-pump surgery in Beating Heart Against Cardioplegic Arrest Studies 1 (BHACAS 1 and 2): A pooled analysis of two randomized controlled trials. Lancet, 359, pp. 1194-9.

4. Arom, K.V., Flavin, T.F., Emery, R.W., Kshettry, V.R., Janey, P.A., Petersen, R.J., 2006. Safety and efficacy of off-pump coronary artery bypass grafting. Ann Thorac Surg, 69, pp. 704-10.

5. Bowles, B.J., Lee, J.D., Dang, C.R., Taoka, S.N., Johnson, E.W., Lau, E.M., et al., 200I. Coronary artery bypass performed without the use of cardiopulmonary bypass is associated with reduced cerebral microemboli and improved clinical results. Chest, 119, pp. 25-30.

6. Boyd, W.D., Desai, N.D., Del Rizo, D.F., Novick, R.J., McKenzie, F.N., Menkis, A.H., 1999. Off-pump surgery decreases post-operative complications and resource utilization in the elderly. Ann Thorac Surg, 68 , pp. $1490-3$.

7. Cohn, L.H., 2008. Myocardial Revascularization Without Cardiopulmonary Bypass, in Cardiac Surgery in the Adult. 3rd ed., New York: McGraw Hill Medical, pp. 633-52.

8. Consten, E.C., Henny, C.P., Eijsman, L., Dongelmans, D.A., van Oers, M.H., 1996. The routine use of fresh frozen plasma in operations with cardiopulmonary bypass is not justified. J Thorac Cardiovasc Surg, 112, pp.162-7.
9. Favaloro, R.G., Effler, D.B., Groves, L.K., Shelton, W.C., Sones, F.M.Jr., 1970. Direct myocardial revascularization by saphenous vein graft: Present operative techniques and indications. Ann Thorac Surg, 10, pp. 97-111.

10. Fouda, M., 2007. Coronary artery bypass surgery with on-pump beating heart technique. Asian Cardiovasc Thorac Ann, 15, pp. 392-5.

11. Gundry, S.R., Romano, M.A., Shattuck, O.H., Razzouk, A.J., Bailey, L.L., 1998. Seven-year follow-up of coronary artery bypasses performed with and without cardiopulmonary bypass. J Thorac Cardiovasc Surg, 115, pp. 1273-8.

12. Hernandez, F., Cohn, W.E., Baribeau, Y., Tryzelaar, J. F., Charlesworth, D. C., Clough, R. A., 2001. In-Hospital Outcomes of Off-pump Versus On-pump Coronary Artery Bypass Procedure: A Multicenter Experience. Ann Thorac Surg, 72, pp. 1528-34.

13. Hu, S., Zheng, Z., Yuan, X., 2010. Increasing long-term major vascular events and resource consumption in patients receiving off-pump coronary artery bypass grafting. A single-center prospective observational study. Circulation, 121, pp. 1800-8.

14. Ishida, M., Kobayashi, J., Tagusari, O., Bando, K., Niwaya, K., Nakajima, H., et al., 2002. Peri-operative advantage of off-pump coronary artery bypass grafting. Circulation, 66. pp. 795-9.

15. Jones, R.H., Hannan, E.L., Hammermeister, K.E., 1996. Identification of preoperative variables needed for risk adjustment of short-term mortality after coronary artery bypass graft surgery: The Working Group Panel on the Cooperative CABG Database Project. J Am Coll Cardiol, 28, p. 1478.

16. Lucchetti, V., Capasso, F., Caputo, M., 1999. Intracoronary shunt prevents left ventricular function impairment during beating heart coronary revascularization. Eur J Cardiothorac Surg, 15, pp. 255-9.

17. Mack, M.J., Pfister, A., Bachand, D., Emery, R., Magee, M.J., Connolly, M., et al., 2004.Comparison of coronary artery bypass surgery with and without cardiopulmonary bypass in patients with multivessel disease. J Thorac Cardiovasc Surg, 127, pp. 167-73.

18. Mathison, M., Edgerton, J.R., Horswell, J.L., Akin, J.J., Mack, M.J., 2000. Analysis of hemodynamic changes during beating heart surgical procedures. Ann Thorac Surg, 70, pp. 1355-61.

19. Meharwal, Z.S., Mishra, Y.K., Kohli, V., Singh, N., Bapna, R.K., Mehta, Y., et al., 2003. Multivessel Off-Pump Coronary Artery Bypass: Analysis of 4953 Cases. The Heart Surgery Forum, 6 (3), pp. 153-9.

20. Puskas, J.D., Wright, C.E., Ronson, R.S., Brown, W.M., Gott, J.P., Guyton, R.A., 1998. Off-pump multivessel coronary bypass via sternotomy is safe and effective. Ann Thorac Surg, 66, pp. 1068-72. 
21. Raja, S.G., Siddiqui, H., Ilsley, C.D., Armani, M., 2009. In-Hospital Outcomes of Off-pump Multivessel Total Arterial and Conventional Coronary Artery Bypass Grafting: Single Surgeon, Single Center Experience. Ann Thorac Surg, 88, pp. 47-53.

22. Roy, A., Stanbridge, R.L., O'Regan, D., 2001. Progression to $100 \%$ offpump coronary artery bypass with the Octopus 1 dual holder. Heart Surg Forum, 4, pp. 174-8.

22. Royse, C.F., Royse, A.G., Wong, C.T., 2003. Assessment of left ventricular function during off-pump coronary artery bypass surgery. Ann Thorac Cardiovasc Surg, 19, pp. 371-7.

23. Ruzzeh, S.A., Ambler, G., Asimakopoulos, G., Omar, R.Z., Hasan, R., Farbi, B., et al., 2003. Off-Pump Coronary Artery Bypass (OPCAB) Surgery Reduces Risk-Stratified Morbidity and Mortality: A United Kingdom Multi-Center Comparative Analysis of Early Clinical Outcome. Circulation, 108[suppl II], pp. II-1-II-8.
24. Sajja, L.R., Mannam, G., Dandu, S.B.R., Pathuri, S.N., Saikiram, K.V.S.S.S., Sompalli, S., 2008. Off-pump coronary artery bypass grafting in patients with significant left ventricular dysfunction. Ind J Thorac Cadiovasc Surg, 24, pp. 110-5.

25. Shroyer, A.L., Grover, F.L., Hattler, B., Collins, J.F., McDonald, G.O., Kozora, E., et al., 2009. On-Pump versus Off-Pump Coronary-Artery Bypass Surgery. N Eng J Med, 361(19), pp. 1827-37.

26. Takai, H., Kobayashi, J., Tagusari, O., Bando, K., Niwaya, K., Nakajima, H., 2006. Off-pump coronary artery bypass grafting for acute myocardial infarction. Circulation, 70, pp. 1303-6.

27. Wan, Y.P., Arifi, A., Wan, S., Yip, J., Thung, K.H., Wong, E, et al., 2004. Beating heart revascularization with or without cardiopulmonary bypass: Evaluation of inflammatory response in a prospective randomized study. J Thorac Cardiovasc Surg, 127, pp. 1624-33.

28. Youn, Y.N., Chang, B.C., Hong, Y.S., Kwak, Y.L., Yoo, K.J., 2007. Early and mid-term impacts of cardiopulmonary bypass on coronary artery bypass grafting in patients with poor left ventricular dysfunction: A propensity score analysis. Circulation, 71, pp. 1387-94. 